\author{
R. Saneifard ${ }^{1^{*}}$ and Nader Hassasi ${ }^{2}$ \\ ${ }^{1}$ Department of Mathematics, Urmia Branch, Islamic Azad University, Oroumieh, Iran. \\ ${ }^{2}$ Department of Mathematics, Malayer Branch, Islamic Azad University, Malayer, Iran.
}

Accepted 12 July, 2013

\begin{abstract}
In the present paper, the researchers discuss the problem of parametric interval approximation of fuzzy numbers. It is the interval which fulfills two conditions. In the first, this interval is a continuous interval approximation operator. In the second, the parametric weighted distance between this interval and the approximated number is minimal and continuous. This interval can be used as a crisp set approximation with respect to a fuzzy quantity. These indices can be applied for comparison of fuzzy numbers namely fuzzy correlations in fuzzy environments and expert's systems. Finally, some of their applications are mentioned.
\end{abstract}

Key words: Fuzzy numbers, defuzzification, interval approximation, correlation.

\title{
INTRODUCTION
}

Representing fuzzy numbers by proper intervals is an interesting and important problem. An interval representation of a fuzzy number may have many useful applications. By using such a representation, it is possible to apply in fuzzy number approaches some results derived in the field of interval number analysis. For example, it may be applied to a comparison of fuzzy numbers by using the order relations defined on the set of interval numbers. Various authors in [Grzegorzewski, 2002; Saneifard, 2009] have studied the crisp approximation of fuzzy sets. They proposed a rough theoretic definition of that crisp approximation, called the nearest interval approximation of a fuzzy set. Moreover, quite different approach to crisp approximation of fuzzy sets was applied in [Cheng et al., 1998]. They proposed a rough theoretic definition of that crisp approximation, called the nearest ordinary set of a fuzzy set, and they suggested a construction of such a set. They discussed rather discrete fuzzy sets. Their approximation of the given fuzzy set is not unique. Thus, this article will not discuss this method. Having reviewed the previous interval approximation, this article proposes here a method to find the parametric interval approximation of a fuzzy number, that, it fulfills two conditions. In the first, this interval is a continuous interval approximation operator. In the second, the parametric distance between this interval and the approximated number is minimal and continuous. The main purpose of this article is for this parametric interval of a fuzzy number be used as a crisp set approximation of a fuzzy number. Some of their applications are mentioned. The paper is organized as follows: In Section 2, this article recalls some fundamental results on fuzzy numbers. In Section 3, a crisp set approximation of a fuzzy number (parametric interval approximate) is obtained. In this Section some remarks are proposed and illustrated. Applications of this approximation are in the Section 4. The paper ends with conclusions in Section 5.

\section{Basic definitions and notations}

The basic definitions of a fuzzy number are given in [Zimmermann, 1991; Saneifard, 2009; Ezzati and 
Saneifard, 2010; Saneifard et al., 2007] as follows:

Definition 1. Let $X$ be a universe set. A fuzzy set $A$ of $X$ is defined by a membership function $\mu_{A}(x) \rightarrow[0,1]$, where $\mu_{A}(x), \forall x \in X$, indicates the degree of $x$ in $A$.

Definition 2. A fuzzy subset $A$ of universe set $X$ is normal if $\operatorname{Sup}_{x \in A} \mu_{A}(x)=1$, where $X$ is the universe set.

Definition 3. A fuzzy subset $A$ of universe set $X$ is convex if $\quad \mu_{A}(\lambda x+(1-\lambda) y) \geq\left(\mu_{A}(x) \wedge \mu_{A}(y)\right)$, $\forall x, y \in X, \forall \lambda \in[0,1]$. In this article symbols $\wedge$ denotes the minimum operators.

Definition 4. A fuzzy set $A$ is a fuzzy number if $A$ is normal and convex on $X$.

Definition 5. For fuzzy set $A$, support function is defined as $\operatorname{Supp}(A)=\left\{x \mid \mu_{A}(x)>0\right\}$, where $\left\{x \mid \mu_{A}(x)>0\right\}$ is closure of set $\left\{x \mid \mu_{A}(x)>0\right\}$.

A space of all fuzzy numbers will be denoted by $F$, and this article recalls that $\operatorname{Core} A=\left\{x \mid \mu_{A}(x)=1\right\}$.

Definition 6. Assume that the fuzzy number $A \in F$ is represented by means of the following representation:

$$
A=\bigcup_{\alpha \in[0,1]}\left(\alpha, A_{\alpha}\right),
$$

Here,

$$
A_{\alpha}=\left\{x: \mu_{A}(x) \geq \alpha\right\} \text {, }
$$

is the $\alpha$-level set of the fuzzy number $A$. This article considers normal and convex fuzzy numbers. Therefore the $\alpha$-level sets may be represented in the form of a segment,

$\forall \alpha \in[0,1]: A_{\alpha}=\left[L_{A}(\alpha), R_{\alpha}(\alpha)\right] \subset(-\infty,+\infty)$

Here, $\quad L:[0,1] \rightarrow(-\infty,+\infty)$ is a monotonically nondecreasing and $R:[0,1] \rightarrow(-\infty,+\infty)$ is a monotonically non-increasing left-continuous functions. The functions $L($.) and $R($.) express the left and right sides of a fuzzy number, respectively. In other words,

$$
L(\alpha)=\mu_{\uparrow}^{-1}(\alpha), R(\alpha)=\mu_{\downarrow}^{-1}(\alpha),
$$

Where $L(\alpha)=\mu_{\uparrow}^{-1}(\alpha)$ and $R(\alpha)=\mu_{\downarrow}^{-1}(\alpha)$ denotes quasiinverse functions of the increasing $g$ and decreasing parts of the membership functions $\mu(x)$, respectively. As a result, the decomposition representation of the fuzzy number $A$, called the $L-R$ representation, has the following form:

$$
A=\bigcup_{\alpha \in[0,1]}\left(\alpha,\left[L_{A}(\alpha), R_{A}(\alpha)\right]\right) \text {. }
$$

Definition 7. [Nasibov, 2007] The following values constitute the weighted averaged representative and weighted width, respectively, of the fuzzy number $A$ :

$$
I(A)=\int_{0}^{1}\left(c L_{A}(\alpha)+(1-c) R_{A}(\alpha)\right) p(\alpha) d \alpha,
$$

and

$$
D(A)=\int_{0}^{1}\left(R_{A}(\alpha)-L_{A}(\alpha)\right) p(\alpha) d \alpha,
$$

Here $0 \leq c \leq 1$ denotes an "optimism/pessimism" coefficient in conducting operations on fuzzy numbers. The function $p:[0,1] \rightarrow[0,+\infty)$ denotes the distribution density of the importance of the degrees of fuzziness, where $\int_{0}^{1} p(\alpha) d \alpha=1$. In particular cases, it may be assumed that $p(\alpha)=(k+1) \alpha^{k}, k=0,1, \cdots$.

Definition 8. For arbitrary fuzzy numbers $A$ and $B$ the quantity

$d_{b}(A, B)=\sqrt{[I(A)-I(B)]^{2}+[D(A)-D(B)]^{2}}$,

is called the parametric distance between the fuzzy numbers $A$ and $B$.

Definition 9. [Grzegorzewski, 2002] An operator $I: F \rightarrow($ Set of Closed Intervalsin $\Re)$ is called an interval approximation operator if for any $A \in F$

$\left(a^{\prime}\right) I(A) \subseteq \operatorname{Supp}(A)$,

$\left(b^{\prime}\right) \operatorname{core}(A) \subseteq I(A)$,

$\left(c^{\prime}\right) \forall(\varepsilon>0) \exists(\delta>0)$ s.t $d(u, v)<\delta \Rightarrow d(I(u), I(v))<\varepsilon$, where $d: F \rightarrow[0,+\infty[$, is a metric defined in the family of all fuzzy numbers.

Definition 10. [Grzegorzewski, 2002]. An interval approximation operator satisfying in condition $\left(c^{\prime}\right)$ for any $A, B \in F$ is called the continuous interval approximation operator.

\section{Parametric interval approximation}

Various authors [Cheng et al., 1998; Grzegorzewski, 
2002] have studied the crisp approximation of fuzzy sets. They proposed a rough theoretic definition of that crisp approximation, called the nearest ordinary set and nearest interval approximation of a fuzzy set. In this Section, the researchers will propose another approximation called the parametric interval approximation. Let $A$ be an arbitrary fuzzy number and $\left[L_{A}(\alpha), R_{A}(\alpha)\right]$ be its $\alpha$-cut set. This article will try to find a closed interval $C_{d_{p}}(A)$, which is the parametric interval to $A$ with respect to metric $d_{p}$. Since each interval with constant $\alpha$-cuts for all $\alpha \in(0,1]$ is a fuzzy number, hence, suppose $C_{d_{p}}(A)=\left[L_{C}, R_{C}\right]$, i.e. $C_{d_{p}}(A)=\left[L_{C}, R_{C}\right], \forall \alpha \in(0,1]$. So, this article has to minimize,

$$
d_{p}\left(A, C_{d_{p}}(A)\right)=\left(\left[I(A)-I\left(C_{d_{p}}(A)\right)\right]^{2}+\left[D(A)-D\left(C_{d_{p}}(A)\right)\right]^{2}\right)^{\frac{1}{2}},
$$

with respect to $L_{C}$ and $R_{C}$, where

$I\left(C_{d_{p}}(A)\right)=\int_{0}^{1}\left(c L_{C}+(1-c) R_{C}\right) p(\alpha) d \alpha, D\left(\left(C_{d_{p}}(A)\right)\right)=\int_{0}^{1}\left(R_{C}-L_{C}\right) p(\alpha) d \alpha$.

In order to minimize $d_{p}$ it suffices to minimize

$\bar{D}_{p}\left(L_{C}, R_{C}\right)=d_{p}^{2}\left(L_{C}, R_{C}\right)$

It is clear that, the parameters $L_{C}$ and $R_{C}$ which minimize Eq. (7) must satisfy

$\nabla \bar{D}_{p}\left(L_{C}, R_{C}\right)=\left(\frac{\partial \bar{D}_{p}}{\partial L_{C}}, \frac{\partial \bar{D}_{p}}{\partial R_{C}}\right)=0$.

Therefore, this article has the following equations:

$$
\left\{\begin{array}{l}
\frac{\partial \bar{D}_{p}\left(L_{C}, R_{C}\right)}{\partial L_{C}}=-2 c \int_{0}^{1}\left(c\left(L_{A}(\alpha)-L_{C}\right)+(1-c)\left(R_{A}(\alpha)-R_{C}\right)\right) p(\alpha) d \alpha \\
+2 \int_{0}^{1}\left(\left(R_{A}(\alpha)-R_{C}\right)-\left(L_{A}(\alpha)-L_{C}\right)\right) p(\alpha) d \alpha=0, \\
\frac{\partial \bar{D}_{p}\left(L_{C}, R_{C}\right)}{\partial R_{C}}=-2(1-c) \int_{0}^{1}\left(c\left(L_{A}(\alpha)-L_{C}\right)+(1-c)\left(R_{A}(\alpha)-R_{C}\right)\right) p(\alpha) d \alpha \\
-2 \int_{0}^{1}\left(\left(R_{A}(\alpha)-R_{C}\right)-\left(L_{A}(\alpha)-L_{C}\right)\right) p(\alpha) d \alpha=0,
\end{array}\right.
$$

The parameters $L_{C}$ associated with the left bound and $R_{C}$ associated with the right bound of the parametric interval can be found by using Eq. (8) as follows:

$$
\left\{\begin{array}{l}
L_{C}=\int_{0}^{1} L_{A}(\alpha) p(\alpha) d \alpha, \\
R_{C}=\int_{0}^{1} R_{A}(\alpha) p(\alpha) d \alpha .
\end{array}\right.
$$

\section{Remark 1}

Since,

$\operatorname{det}\left[\begin{array}{ll}\frac{\partial^{2} \bar{D}_{p}\left(L_{C}, R_{C}\right)}{\partial L_{C}^{2}} & \frac{\partial^{2} \bar{D}_{p}\left(L_{C}, R_{C}\right)}{\partial R_{C} \partial L_{C}} \\ \frac{\partial^{2} \bar{D}_{p}\left(L_{C}, R_{C}\right)}{\partial L_{C} \partial R_{C}} & \frac{\partial^{2} \bar{D}_{p}\left(L_{C}, R_{C}\right)}{\partial R_{C}^{2}}\end{array}\right]=\operatorname{det}\left[\begin{array}{cc}2 c^{2} & 2 c(1-c)-2 \\ 2 c(1-c)-2 & 2(1-c)^{2}+2\end{array}\right]=4>0$, and $\forall c \in[0,1], \frac{\partial \bar{D}_{p}^{2}\left(L_{C}, R_{C}\right)}{\partial L_{C}^{2}}=1>0$, therefore $L_{C}$ and $R_{C}$ given by (9), minimize $d_{p}\left(A, C_{d_{p}}(A)\right)$. Therefore, the interval

$C_{d_{p}}(A)=\left[\int_{0}^{1} L_{A}(\alpha) p(\alpha) d \alpha, \int_{0}^{1} R_{A}(\alpha) p(\alpha) d \alpha\right]$,

is the nearest parametric interval approximation of fuzzy number $A$ with respect to metric $d_{p}$.

\section{Remark 2}

Whenever, in distribution density function $p(\alpha)=(k+1) \alpha^{k}$, assume that $k=1$, therefore $C_{d_{p}}(A)$ is weighted intervalvalue possibilistic mean [6], (Carlsson and Full'er, 2001).

\section{Remark 3}

If, in distribution density function $p(\alpha)=(k+1) \alpha^{k}$, assume $k=0$, therefore $C_{d_{p}}(A)$ is expected interval [10].

However, this article wants to approximate a fuzzy number by a crisp interval. Thus, the researchers have to use an operator $C_{d_{p}}(A): F \rightarrow($ Setof Closed Intervalsin $\Re)$ which transforms fuzzy numbers into family of closed intervals on the real line.

\section{Theorem 1}

The operator $C_{d_{p}}(A): F \rightarrow($ Setof Closed Intervalsin $\Re)$ is an interval approximation operator, i.e. $C_{d_{p}}(A)$ is a continuous interval approximation operator. 


\section{Proof}

It is easy to verify that the conditions $\left(a^{\prime}\right)$ and $\left(b^{\prime}\right)$ are held. For the Proof of $\left(c^{\prime}\right)$, let $A$ and $B$ be two fuzzy numbers with parametric intervals $C_{d_{p}}(A)=\left[L_{C}(A), R_{C}(A)\right]$ and $C_{d_{p}}(B)=\left[L_{C}(B), R_{C}(B)\right]$, respectively. Then

$$
\begin{aligned}
& d^{2}{ }_{p}\left(C_{d_{p}}(A), C_{d_{p}}(B)\right)=\left\lfloor I\left(C_{d_{p}}(A)\right)-I\left(C_{d_{p}}(B)\right)\right]^{2}+\left\lfloor D\left(C_{d_{p}}(A)\right)-D\left(C_{d_{p}}(B)\right)\right\rfloor^{2} \\
& =\left[\int_{0}^{1}\left(c L_{C}(A)+(1-c) R_{C}(A)\right) p(\alpha) d \alpha-\int_{0}^{1}\left(c L_{C}(B)+(1-c) R_{C}(B)\right) p(\alpha) d \alpha\right]^{2}
\end{aligned}
$$$$
+\left[\int _ { 0 } ^ { 1 } \left(\left(R_{C}(A)+L_{C}(A)\right) p(\alpha) d \alpha-\int_{0}^{1}\left(\left(R_{C}(B)+L_{C}(B)\right) p(\alpha) d \alpha\right]^{2}\right.\right.
$$$$
=\left[c\left(L_{C}(A)-L_{C}(B)\right)+(1-c)\left(R_{C}(A)-R_{C}(B)\right)\right]^{2}+\left[\left(R_{C}(A)-R_{C}(B)\right)-\left(L_{C}(A)-L_{C}(B)\right)\right]^{2}
$$$$
=\left[c\left(\int_{0}^{1} L_{A}(\alpha) p(\alpha) d \alpha-\int_{0}^{1} L_{B}(\alpha) p(\alpha) d \alpha\right)+(1-c)\left(\int_{0}^{1} R_{A}(\alpha) p(\alpha) d \alpha-\int_{0}^{1} R_{B}(\alpha) p(\alpha) d \alpha\right)\right]^{2}
$$$$
+\left[\left(\int_{0}^{1} R_{A}(\alpha) p(\alpha) d \alpha-\int_{0}^{1} R_{B}(\alpha) p(\alpha) d \alpha\right)+\left(\int_{0}^{1} L_{A}(\alpha) p(\alpha) d \alpha-\int_{0}^{1} L_{B}(\alpha) p(\alpha) d \alpha\right)\right]^{2}
$$$$
=\left[c \int_{0}^{1}\left(L_{A}(\alpha)-L_{B}(\alpha)\right) p(\alpha) d \alpha+(1-c) \int_{0}^{1}\left(R_{A}(\alpha)-R_{B}(\alpha)\right) p(\alpha) d \alpha\right]^{2}
$$$$
+\left[\int_{0}^{1}\left(R_{A}(\alpha)-R_{B}(\alpha)\right) p(\alpha) d \alpha-\int_{0}^{1}\left(L_{A}(\alpha)-L_{B}(\alpha)\right) p(\alpha) d \alpha\right]^{2}=d_{p}^{2}(A, B) .
$$

It means that $\forall \varepsilon>0, \exists \delta=\varepsilon>0$, when $d_{p}(A, B)<\delta$, then, this article has $d_{p}\left(C_{d_{p}}(A), C_{d_{p}}(B)\right)<\varepsilon$. It shows that our parametric interval approximation is continuous interval approximation. In other words, if $A$ and $B$ are close enough, then their parametric interval approximations obtained by $C_{d_{p}}$ are also close enough.

\section{Application}

In this section, the researchers introduce some of applications of the weighted interval approximation of fuzzy numbers. These indices can be applied for comparison of fuzzy numbers namely fuzzy correlations in fuzzy environments and expert's systems.

\section{Correlation between fuzzy numbers}

In many applications the correlation between fuzzy numbers is of interest. Several authors have proposed different measures of correlation between membership functions, intuitionistic fuzzy sets and correlation [Carlsson et al., 2005; Bustince and Burillo, 1995]. Hung and Wu [2002] defined a correlation by means of expected interval. They defined the correlation coefficient between fuzzy numbers $A$ and $B$ as follows:

$$
\rho(A, B)=\frac{E_{*}(A) E_{*}(B)+E^{*}(A) E^{*}(B)}{\sqrt{E_{*}^{2}(A)+E^{* 2}(A)} \sqrt{E_{*}^{2}(B)+E^{* 2}(B)}},
$$

where

$$
\left[E_{*}(A), E^{*}(B)\right]=\left[\int_{0}^{1} L_{A}(\alpha) d \alpha, \int_{0}^{1} R_{A}(\alpha) d \alpha\right] .
$$

This correlation coefficient shows not only the degree of relationship between the fuzzy numbers but also whether these fuzzy numbers are positively or negatively related. The researchers extend (11) by interval $C_{d_{p}}(A)=\left[L_{C}, R_{C}\right]$ instead of (12), then

$$
\rho_{p}(A, B)=\frac{L_{C}(A) L_{C}(B)+R_{C}(A) R_{C}(B)}{\sqrt{L_{C}^{2}(A)+R_{C}^{2}(A)} \sqrt{L_{C}^{2}(B)+R_{C}^{2}(B)}} .
$$

$\rho_{p}(A, B)$ is called the weighted correlation coefficient between two fuzzy numbers $A$ and $B$. This correlation coefficient lies in $[-1,1]$ and gives us more information compared to correlation coefficient in [Sezer, 2012; Zimmermann, 1991] and some others, which lie within $[0,1]$. It has all mentioned properties from correlation coefficient introduced by Zimmermann [1991]; the researchers review properties $\rho_{p}(A, B)$ as follows:

\section{Proposition}

For any fuzzy numbers $A$ and $B$, we have:

1. $\rho_{p}(A, B)=\rho_{P}(B . A)$.

2. If $A=B$ then $\rho_{p}(A, B)=1$.

3. If $A=c B$, for some $c>0 \Rightarrow \rho(A, B)=1$.

4. $\left|\rho_{p}(A, B)\right| \leq 1$.

\section{Proof}

The proof is obvious.

\section{Example}

Let $A_{\alpha}=\left[a_{1}+\left(a_{2}-a_{1}\right) \alpha, a_{4}-\left(a_{4}-a_{3}\right) \alpha\right] \quad$ and 
Table 1. Summary of the result of comparison.

\begin{tabular}{lccccc}
\hline Method & $\rho_{p}\left(G_{6}, G_{7}\right)$ & $\rho_{p}\left(G_{6}, G_{8}\right)$ & $\rho_{p}\left(G_{6}, G_{9}\right)$ & $\rho_{p}\left(G_{6}, G_{10}\right)$ & $\rho_{p}\left(G_{6}, G_{11}\right)$ \\
\hline Hong and Wang & 0.852 & 0.778 & 0.778 & 0.778 & 0.857 \\
Murty et. al & 0.667 & 0.500 & 0.500 & 0.500 & 0.500 \\
Sahnoun et. al & 0.224 & 0.000 & 0.000 & 0.000 & 0.000 \\
Proposed method with k=1 & 0.949 & 0.857 & 0.814 & 0.789 & 0.743 \\
Proposed method with k=2 & 0.894 & 0.814 & 0.781 & 0.763 & 0.731 \\
\hline
\end{tabular}

$B_{\alpha}=\left[b_{1}+\left(b_{2}-b_{1}\right) \alpha, b_{4}-\left(b_{4}-b_{3}\right) \alpha\right]$ be two trapezoidal fuzzy numbers and $p(\alpha)=(k+1) \alpha^{k}, k=1,2, \cdots$ is the weighted function. Then,

$\rho_{p}(A, B)=\frac{\left((k+1) a_{2}+a_{1}\right)\left((k+1) b_{2}+b_{1}\right)+\left((k+1) a_{3}+a_{4}\right)\left((k+1) b_{3}+b_{4}\right)}{\sqrt{\left((k+1) a_{2}+a_{1}\right)^{2}+\left((k+1) a_{3}+a_{4}\right)^{2}} \sqrt{\left((k+1) b_{2}+b_{1}\right)^{2}+\left((k+1) b_{3}+b_{4}\right)^{2}}}$.

The above example shows that the parametric function interacts on the correlation coefficient between two fuzzy numbers such that for large values of $k$ this article has:

$\lim _{k \rightarrow \infty} \rho_{p}(A, B)=\frac{a_{2} b_{2}+a_{3} b_{3}}{\sqrt{a_{2}^{2}+a_{3}^{2}} \sqrt{b_{2}^{2}+b_{3}^{2}}}$

Moreover if $A$ and $B$ be two triangular fuzzy numbers, whenever $k \rightarrow \infty$, we have

$\rho_{p}(A, B)=\frac{a_{2} b_{2}}{\left|a_{2} b_{2}\right|}, a_{2}, b_{2} \neq 0$.

\section{Example}

Yen [2012] used to six grade levels (6th-grade to 11thgrade) as student's mathematical learning progress. He assigned the linguistic values $G_{6}, G_{7}, G_{8}, G_{9}, G_{10}$ and $G_{11}$ to these grade levels, respectively, and transferred these linguistic values to corresponding reasonable normal fuzzy numbers $G_{6}, G_{7}, G_{8}, G_{9}, G_{10}$ and $G_{11}$ with triangular membership functions as follows:

$$
\begin{aligned}
& \mu_{G_{6}}= \begin{cases}1-5 x, & 0 \leq x \leq 0.2 \\
0, & 0.2 \leq x \leq 1\end{cases} \\
& \mu_{G_{7}}= \begin{cases}5 x, & 0 \leq x \leq 0.2 \\
2-5 x, & 0.2 \leq x \leq 0.4, \\
0, & 0.4 \leq x \leq 1\end{cases} \\
& \mu_{G_{8}}= \begin{cases}0, & 0 \leq x \leq 0.2 \\
5 x-1, & 0.2 \leq x \leq 0.4 \\
3-5 x, & 0.4 \leq x \leq 0.6 \\
0, & 0.6 \leq x \leq 1\end{cases} \\
& \mu_{G_{9}}=\left\{\begin{array}{ll}
0, & 0 \leq x \leq 0.4, \\
5 x-2, & 0.4 \leq x \leq 0.6, \\
4-5 x, & 0.6 \leq x \leq 0.8, \\
0, & 0.8 \leq x \leq 1 .
\end{array} \quad \mu_{G_{10}}= \begin{cases}0, & 0 \leq x \leq 0.6, \\
5 x-3, & 0.6 \leq x \leq 0.8, \\
5-5 x, & 0.8 \leq x \leq 1 .\end{cases} \right. \\
& \mu_{G_{11}}= \begin{cases}0, & 0 \leq x \leq 0.8, \\
5 x-4, & 0.8 \leq x \leq 1 .\end{cases}
\end{aligned}
$$

The researchers use the parametric correlation coefficient to compute $\rho_{p}\left(G_{6}, G_{i}\right), i=7,8,9,10,11$. The result of comparison is summarized in Table 1. Throughout this section the researchers assumed that $p(\alpha)=(k+1) \alpha^{k}$.

This example shows that $\rho_{p}\left(G_{6}, G_{i}\right)$ is decreasing in $i$, intui-tively. But the methods of [Wang and Li, 2007; Bustince and Burillo, 1995; Yu, 1993] have no decreasing behavior. For our method weighted function $f$ interacts on correlation value between two fuzzy numbers. Hence, there is reasonable advantage in using the proposed formula (13).

\section{Conclusion}

In this study, the researchers introduce the nearest weighted interval approximation of a fuzzy number. A new type of correlation coefficient between fuzzy numbers is proposed and it is compared with some other methods. These indices can be used in many times series and regression models in a fuzzy environment.

\section{REFERENCES}

Bustince H, Burillo P (1995). Correlation of interval-valued intuitionistic fuzzy sets, Fuzzy Sets Syst. 74:237-244.

Carlsson C, Full'er R (2001). On possibilistic mean value and variance of fuzzy numbers, Fuzzy Sets Syst. 122:315-326.

Carlsson C, Full'er R, Majlender P (2005). On possibilistic correlation, Fuzzy Sets Syst. 155:425-445.

Cheng $\mathrm{CH}$, Biswas R, Nanda S (1998). Nearest ordinary set of a fuzzy set: a rough theoretic construction, Bull. Polish Acad. Sci. 46:105114.

Ezzati R, Saneifard R (2010). A new approach for ranking of fuzzy numbers with continuous weighted quasi-arithmetic means, Math. Sci. 4:143-158.

Grzegorzewski P (2002). Nearest interval approximation of a fuzzy number, Fuzzy Sets Syst. 130:321-330.

Hung W, Wu J (2002). Correlation of intuitionistic fuzzy sets by centroid method, Inform. Sci. 144:219 - 225.

Nasibov EN (2007). Fuzzy least squares regression model based of weighted distance between fuzzy numbers, Automatic Control Comput. Sci. 41:10- 17.

Saneifard R (2009). A method for defuzzification by weighted distance, Int. J. Ind. Math. 3:209-217.

Saneifard R, Allahviranloo T, Hosseinzadeh F, Mikaeivand N (2007). Euclidean ranking DMU's with fuzzy data in DEA, A.M.S. 60:29892998.

Sezer S (2012). Using fuzzy sets in developing mathematical learningprogress indicator, Proc. Natl. Sci. Coune. ROC (D) 6. 
Wang ZX, Li H (2007). Weighted triangular approximation of fuzzy numbers, Int. J. Approximate Reason. 46:137-150.

Yu C (1993). Correlation of fuzzy numbers, Fuzzy Sets Syst. 55:303307.
Zimmermann HJ (1991). Fuzzy sets theory and its applications, Kluwer Academic Press, Dordrecht, 1991. 